Article

\title{
Pharmaceutical Efficacy of Gypenoside LXXV on Non-Alcoholic Steatohepatitis (NASH)
}

\author{
Jin Ha Lee ${ }^{1,+} \mathbb{\oplus}$, Ji Young $\mathrm{Oh}^{2,+}$, Soo Hyun Kim ${ }^{2}$, In Jeong $\mathrm{Oh}^{2}$, Yong-ho Lee ${ }^{2}{ }^{\mathbb{D}}$, \\ Keun Woo Lee ${ }^{1}$, Woong Hee Lee ${ }^{1,3}$ and Jeong-Hwan Kim ${ }^{1,4, *}$ \\ 1 MODNBIO Inc., digital road 34, Kolon Science Valley I, Guro-gu, Seoul 08378, Korea; \\ jinha1118@modnbio.com (J.H.L.); leekw@modnbio.com (K.W.L.); xpress24@nate.com (W.H.L.) \\ 2 Department of Internal Medicine, Yonsei University College of Medicine, 50-1 Yonsei-ro, Seodaemun-gu, \\ Seoul 03722, Korea; iamojy@gmail.com (J.Y.O.); shkim6784@naver.com (S.H.K.); \\ olib3068@gmail.com (I.J.O.); YHOLEE@yuhs.ac (Y.-h.L.) \\ 3 Institute of Biotechnology, Chungnam National University, Daejeon 34134, Korea \\ 4 Cardiovascular Research Institute, Graduate School of Medicine, Yokohama City University, 3-9 Fukuura, \\ Kanazawa-ku, Yokohama 236-0004, Japan \\ * Correspondence: jhk@modnbio.com \\ + These authors contributed equally to this work.
}

Received: 1 September 2020; Accepted: 7 October 2020; Published: 8 October 2020

check for updates

\begin{abstract}
Ginsenosides have offered a wide array of beneficial roles in the pharmacological regulation of hepatic metabolic syndromes, including non-alcoholic steatohepatitis (NASH), non-alcoholic fatty liver disease (NAFLD), and obesity. Of the numerous ginsenosides, Rg3 has been widely investigated, but there have been few studies of gypenosides (Gyp). Particularly, no study on Gyp LXXV has been reported to date. Here, to firstly explore the pharmacological effects of Gyp LXXV against NASH and the related mechanism, methionine- and choline-deficient (MCD) diet-induced NASH mice and hepatic cells (stellate cells, hepatic macrophages, and hepatocytes) were selected. Gyp LXXV exhibited markedly alleviated MCD diet-induced hepatic injury, inflammation, and fibrosis by down-regulating hepatic fibrosis markers such as $\alpha$-smooth muscle actin $(\alpha$-SMA), collagen1, transforming growth factors- $\beta$ (TGF- $\beta 1$ ), tumor necrosis factor- $\alpha$ (TNF- $\alpha$ ), MCP- 1 , interleukin (IL)- $1 \beta$, nuclear factor $\mathrm{KB}(\mathrm{NF} \mathrm{KB})$, and GRP78. Remarkably, histopathological studies confirmed that $15 \mathrm{mg} / \mathrm{kg}$ of Gyp LXXV administration to MCD diet-induced mice led to effective prevention of liver injury, lipid accumulation, and activation of hepatic macrophages, indicating that Gyp LXXV might be a potential anti-NASH drug.
\end{abstract}

Keywords: ginsenosides; gypenoside LXXV; drug discovery; liver fibrosis; nonalcoholic steatohepatitis (NASH); hepatic stellate cells

\section{Introduction}

Non-alcoholic steatohepatitis (NASH) is a severe liver disease that begins with fat accumulation in the organ, resulting in chronic liver injury, necrosis, inflammation, and unbalanced lipid metabolism in the liver, which can lead to hepatic fibrosis, dysfunction, and even cirrhosis which ultimately leads to cancer [1-9]. Particularly, obesity and insulin resistance are considered main factors in the initiation and perpetuation of NASH [10] and play a key role in the pathogenesis of type 2 diabetes mellitus and non-alcoholic fatty liver disease (T2DM-NAFLD) [11].

At the cellular level, liver damage through fat accumulation induces mitochondrial dysfunction, which can cause harmful effects on hepatic inflammation, reactive oxygen species (ROS) homeostasis, and cell death, resulting in NASH [12]. Simultaneously, it activates hepatic stellate cells (HSC) 
via progressive release of pro-inflammatory cytokines (platelet derived growth factor (PDGF), transforming growth factors- $\beta$ (TGF- $\beta$ ), interleukin (IL)-1, tumor necrosis factor- $\alpha$ (TNF- $\alpha$ ), and some chemokines) [13-15]. The activated HSCs multiply fibrogenic cells, generating a micro-environment favorable to cellular proliferation and survival by upregulating mesenchymal markers ( $\alpha$-smooth muscle actin ( $\alpha$-SMA, ACTA2), desmin (DES), and collagen $\alpha 1(\mathrm{I})$ ) [13-15].

TGF- $\beta$ has been considered a major pro-fibrogenic cytokine and a promising target for treating hepatic fibrosis [15-18]. Although there have been many clinical reports focused on the importance of TGF- $\beta$ activation in the pathogenesis of liver fibrosis in patients, its inhibition causes undesirable side effects, thereby decreasing the therapeutic advantages $[15,17,18]$. Therefore, understanding the pleiotropic effects of TGF- $\beta$ and its regulatory mechanisms will help design better TGF- $\beta$-based therapeutics. Although there is no approved cure for NASH and a game-changing clinical approach has not yet been identified, several studies to ameliorate NASH are being performed in clinical trials $[17,18]$. In fact, an emerging trend in NAFLD or NASH treatment is represented by healthy lifestyle modifications, including diet and exercise [19].

As an active ingredient derived from natural products, ginseng ginsenoside has excellent therapeutic effectiveness with high bioavailability and little biotoxicity, and has been widely employed to treat various metabolic syndromes such as diabetes, liver diseases, heart diseases, cancer, etc [20-24]. A mixture of ginsenoside $\mathrm{Rg} 3, \mathrm{Rg} 1, \mathrm{Rb} 1$, and probiotics has been used as a putative treatment for NAFLD symptoms to diminish liver inflammation by decreasing the expression of IL-1 $\beta$ and phospho-p38 [23]. Ginsenoside Rg1 exhibited an anti-inflammatory effect on palmitic acid-induced HepG2 cells via the AMPK/NFkB pathway [24].

Gypenosides (Gyp) are a dammarane-type triterpene glycoside extracted from Gynostemma (G.) pentaphyllum (Thunb.) Makino possesses numerous pharmacological properties on metabolic diseases, including anti-aging, anti-oxidation, anti-inflammation, cardioprotective and neuroprotective effects, as well as wound healing properties [25-27]. Gyp extracts also have been shown to have a therapeutic effect of anti-hepatic lipogenesis [28,29]. Notably, a recent study showed that it alleviated NAFLD in mice by enhancing the intestinal probiotic property [29]. Furthermore, Gyp extracts exhibited a hepato-protective effect on T2DM-NAFLD by down-regulating the mRNA levels of tumor necrosis factor- $\alpha(\mathrm{TNF}-\alpha)$, nuclear factor $\kappa \mathrm{B}(\mathrm{NF} \kappa \mathrm{B})$, peroxisome proliferator activated receptor $(\mathrm{PPAR} \gamma)$, and cytochrome P4501A1 (CYP1A1) [30]. However, there is a limited number of reports and a lack of progress on drug screening and development of Gyp compounds since the regulatory mechanism on liver fat metabolism of Gyp compounds' action in targeting NASH is still uncertain. In this study, for the first time, we deliver compelling evidence on the therapeutic efficacy of a Gyp LXXV (one of the Gyp compound types) compound against high-fat diet-induced NASH, finding the potential mechanisms involved in liver fibrogenesis.

\section{Materials and Methods}

\subsection{Materials}

Fetal bovine serum (FBS), Dulbecco's modified Eagle's medium (DMEM), Roswell Park Memorial Institute (RPMI)-1640 culture medium and penicillin-streptomycin were purchased from GE Healthcare Life Sciences (Marlborough, MA, USA). IL-1 $\beta$ ELISA kit (Human 88-7261-22) and TRIzol reagent (15596018) was purchased from Invitrogen (Carlsbad, CA, USA). Lipopolysaccharide (LPS; L6529), palmitate (P9767) and MCC950 (PZ0280) were obtained from Sigma-Aldrich (St. Louis, MO, USA). Pioglitazone was purchased from Takeda Pharmaceutical (AD-4833, Kanagawa, Japan). Ezetimibe was obtained from Cayman Chemical (16331, Ann Arbor, MI, USA). The triglyceride assay kit was purchased from Bioassay systems (ETGA-200, Hayward, CA, USA). Anti-F4/80 antibody was obtained from Abcam (ab6640, Cambridge, MA, USA). 3-(4,5-dimethylthiazol-2-yl)-2,5-diphenyltetrazolium bromide (MTT) was purchased from Georgiachem (MT 1036, Norcross, GA, USA). 


\subsection{Characterization of High-Purity Gyp LXXV and Ginsenoside Rg3 Compounds}

The Gyp LXXV and ginsenoside Rg3 compounds were prepared at MODNBIO Inc. (Seoul, Korea) and analyzed by HPLC systems that were controlled by LabSolutions ${ }^{\circledR}$ software (Shimadzu, Japan) running under Microsoft Windows 7. Each compound was dissolved in a methanol solution, and $20 \mu \mathrm{L}$ of the solution was injected into the column (ODS, $250 \times 4.6 \mathrm{~mm}, 150 \AA$ ). The flow rate was $1.0 \mathrm{~mL} / \mathrm{min}$, and the UV-vis absorbance peaks were measured at $203 \mathrm{~nm}$.

\subsection{Cell Culture and Viability Assay}

Hepatic cell line HepG2 and hepatic stellate cell line LX2 were cultured with DMEM supplemented with $10 \%$ FBS and 1\% penicillin-streptomycin solution, and they were grown in $100 \mathrm{~mm}$ culture dishes in a $5 \% \mathrm{CO}_{2}$ incubator at $37^{\circ} \mathrm{C}$. THP-1 cells were cultured with RPMI- 1640 culture medium supplemented with $10 \%$ FBS and 1\% penicillin-streptomycin solution and were grown in $75 \mathrm{~cm}^{2}$ flasks. The cytotoxicity in cultured cells was investigated through MTT assay (tetrazolium-based colorimetric). Cells were treated with either Gyp LXXV or Rg3. After treatment, the cells were incubated with $5 \mathrm{mg} / \mathrm{mL}$ MTT reagent for $1 \mathrm{~h}$ at $37^{\circ} \mathrm{C}$ in the dark. MTT reagent was aspirated, and dimethyl sulfoxide was added to each well to dissolve MTT formazan crystals. Absorbance was measured at $560 \mathrm{~nm}$ using a multiple plate reader.

\subsection{ELISA for IL-1 $\beta$-Detection}

After stimulating the THP-1 cells with macrophages, the cultured supernatant was collected, and then IL-1 $\beta$ concentration was determined using an IL-1 $\beta$ ELISA kit (Thermo Fisher Scientific, 88-7261-88) according to the manufacturer's instructions.

\subsection{RNA Isolation and Reverse Transcription-Polymerase Chain Reaction (RT-PCR)}

Total RNA was isolated using TRIzol reagent, and then cDNA was synthesized from the total RNA using the High-Capacity cDNA Reverse Transcription kit (Applied Biosystems, 4368814, Waltham, MA, USA). The cDNA was mixed with the Power SYBR ${ }^{\circledR}$ Green PCR Master Mix (Applied Biosystems, 4367659, Waltham, MA, USA) and primers, and amplified in StepOne ${ }^{\mathrm{TM}}$ Real-Time PCR Systems (Applied Biosystems, Waltham, MA, USA).

\subsection{Animal Preparation}

Seven-week-old male C57BL/6J mice weighing 20-25 g were purchased from Japan SLC, Inc. (Hamamatsu, Shizuoka, Japan). Mice were acclimatized seven days before the start of the experiment in the facility under standard environmental conditions $\left(23 \pm 2{ }^{\circ} \mathrm{C}, 12 / 12 \mathrm{~h}\right.$ light/dark cycle with lights on at 08:00) and had ad libitum access to food and water. All animal care and procedures were performed in accordance with Yonsei University College of Medicine guidelines and approved by the institutional animal care and use committee at Yonsei University College of Medicine (\#2018-0275).

\subsection{Animal NASH Model}

Mice were fed a normal diet (vehicle) or a methionine- and choline-deficient (MCD) L-amino acid diet (Research Diet, Inc., A02082002BR, NJ, USA) for 6 weeks. The mice were randomly divided into seven groups as vehicle, MCD, Rg3 (15, $30 \mathrm{mg} / \mathrm{kg} /$ daily), Gyp LXXV (15, $30 \mathrm{mg} / \mathrm{kg} /$ daily) and MCC950 (positive control). Each sample was orally administrated during the last 3 weeks of the 6 weeks of being fed the MCD diet. After administration of the compounds for 3 weeks, the MCD mice were euthanized through cardiac blood collection; following this, the liver tissue was sampled. 


\subsection{Blood Chemistry Assay}

Serum was isolated from the blood collected from the mice using centrifugation at $3000 \mathrm{rpm}$ for $15 \mathrm{~min}$. Alanine aminotransferase (ALT) (Fujifilm, 3250) and aspartate aminotransferase (AST) (Fujifilm, 3150) in serum were analyzed using Fuji dry-chem 4000i (Fujifilm, Barcelona, Spain).

\subsection{Immunohistochemistry (IHC)}

Paraffin-embedded tissue sections were deparaffinized with xylene, and then rehydrated in serial dilutions of ethanol. The tissues were washed using distilled water and were then heated in antigen retrieval by sodium citrate buffer $(\mathrm{pH}$ 6). The tissues were incubated with hydrogen peroxidase blocking solution (S2023, Dako, Denmark) for $10 \mathrm{~min}$ at room temperature (RT) and were treated with protein blocking solution for $20 \mathrm{~min}$ at RT. The tissues were incubated at $4{ }^{\circ} \mathrm{C}$ overnight with a monoclonal anti-F4/80 antibody (1:100 dilution) or a polyclonal anti- $\alpha$-SMA antibody (1:100 dilution). The slides were incubated for $30 \mathrm{~min}$ with a secondary antibody and then incubated with avidin-biotin complex reagent for $30 \mathrm{~min}$ at RT. The tissues were developed with 3,3'Diaminobenzidine (DAB) substrate and counterstained with hematoxylin. IHC staining intensities were analyzed using Fiji software (National Institutes of Health, Maryland, MA, USA). F4/80- or $\alpha$-SMA-positive cells were normalized by nuclei.

\subsection{Oil Red O Staining}

For frozen sections, liver tissues were fixed with $4 \%$ paraformaldehyde overnight and then infiltrated in $30 \%$ sucrose. The tissues were embedded in OCT compound and stored at $-80{ }^{\circ} \mathrm{C}$ until analysis. Frozen tissues were sectioned at $6 \mu \mathrm{m}$ using a cryostat (CM1860, Leica Biosystems, IL, USA). The sections were air-dried for $30 \mathrm{~min}$ and fixed in 10\% formalin for $5 \mathrm{~min}$. The slides were washed with $60 \%$ isopropanol and then stained with oil red O (Sigma-Aldrich, O0625) working solution for $15 \mathrm{~min}$. The slides were counterstained with hematoxylin and washed with distilled water.

\subsection{Western Blot Analysis}

Cells and liver tissues were lysed with RIPA buffer (ATTO Corporation, Tokyo, Japan) and quantified using a BCA protein assay kit (Thermo Fisher Scientific, Waltham, MA, USA). Proteins were separated by SDS-PAGE and transferred to a polyvinylidene difluoride membrane. The membrane was incubated with 5\% skim milk for blocking, and then washed with Tris-buffered saline, $0.1 \%$ Tween 20 (TBST). The membrane was incubated with primary antibodies against $\alpha$-SMA (Abcam, ab5694), p-NFkB (Cell Signaling, 3033S), NFkB (Santa Cruz, sc-372), Glyceraldehyde 3-phosphate dehydrogenase (GAPDH) (Santa Cruz, sc-25778) overnight at $4{ }^{\circ} \mathrm{C}$. Specific bands were visualized by a Chemi-luminescent Image Analyzer (ImageQuant ${ }^{\mathrm{TM}}$ LAS 4000, Fujifilm, Tokyo, Japan) and analyzed by Image J software.

\subsection{Hepatic Triglyceride (TG) Assay}

After $30 \mathrm{mg}$ of liver tissue was homogenized, the analysis of hepatic TG was performed using the EnzyChrom $^{\mathrm{TM}}$ Triglyceride Assay Kit (ETGA-200, BioAssay Systems, Waltham, CA, USA) according to the manufacturer's instructions.

\subsection{Statistics}

Data were presented as mean \pm standard error of mean (SEM). The results were analyzed by unpaired two-tailed Student's t-test. 


\section{Results and Discussion}

\subsection{Characterization of High-Purity Gyp LXXV and Ginsenoside Rg3 Compounds}

The HPLC chromatogram clearly confirmed the high-quality ( 99\%) single signatures of Gyp LXXV and Rg3 samples (Figure S1). The solubility of the compounds was confirmed using a turbidity method prior to all the in vitro and in vivo studies, as reported in the previous study [31].

\subsection{Gyp LXXV Inhibits the TGF- $\beta$-Induced Activation of Hepatic Stellate Cells}

Throughout chronic liver injury, TGF- $\beta$ plays a prominent role in stimulating liver fibrogenesis by myofibroblast-like cells derived from HSCs. To assess the potential inhibitory effect of Gyp LXXV against TGF- $\beta$-induced HSCs, the in vitro inhibitory rate of the compounds was monitored in LX-2, a well-established HSC cell line. The cell viability of Gyp LXXV in the LX-2 cells was confirmed by MTT assays, showing no cytotoxicity at all concentrations (Figure 1a). To evaluate the regulatory effects of Gyp LXXV in the TGF- $\beta$-induced activation of LX-2, the expressions of fibrosis markers $(\alpha$-SMA and collagen1) were examined by a RT-PCR analysis. In Figure $1 b, c$, following treatment with TGF- $\beta 1$ to induce a fibrosis, the RNA expressions of $\alpha$-SMA and collagen 1 in LX-2 were dramatically increased compared to the control. The Gyp LXXV treatment significantly reduced the production of $\alpha$-SMA and collagen 1 in a dose-dependent manner. The results were similar to those obtained when cells were exposed to $\operatorname{Rg} 3$ used as a ginsenoside control treatment.

(a)

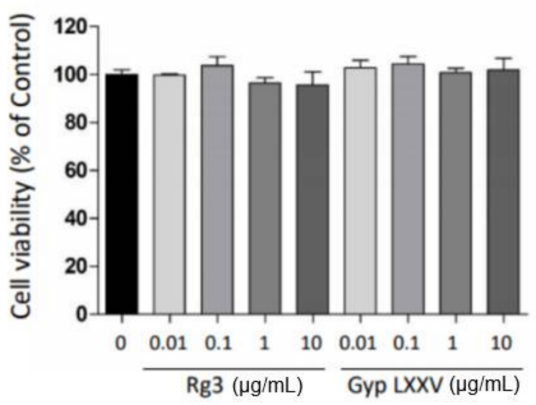

(b)

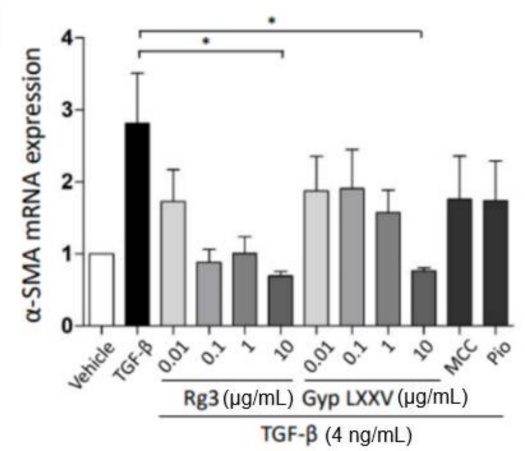

(c)

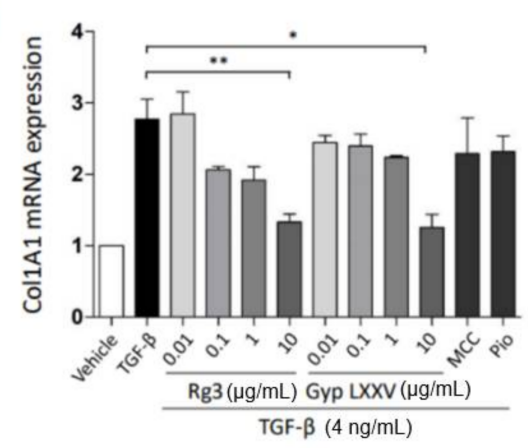

Figure 1. Effect of gypenoside LXXV (Gyp LXXV) on cell viability and fibrosis in hepatic stellate cells (LX-2). Cell viability of Gyp LXXV or Rg3 on LX-2 cells (a). Cells were treated with increasing concentrations of Gyp LXXV or Rg3 $(0.01-10 \mu \mathrm{g} / \mathrm{mL})$ for $24 \mathrm{~h}$. Gyp LXXV-mediated inhibition of fibrosis markers, $\alpha$-smooth muscle actin ( $\alpha$-SMA) (b) and collagen1 (c), via transforming growth factors- $\beta$ (TGF- $\beta$ ) $(4 \mathrm{ng} / \mathrm{mL})$-induced LX-2 activation ( $\mathrm{n}=3$ for each group). ${ }^{*} p<0.05,{ }^{* *} p<0.01 \mathrm{vs.} \mathrm{TGF-} \beta$. MCC950 (MCC; $1 \mu \mathrm{M}$ ) or pioglitazone (Pio; $10 \mu \mathrm{M}$ ) was used as positive controls.

Furthermore, MCC950, a selective inhibitor of NLRP3 inflammasome, has been used to treat the liver inflammation and fibrosis [32]. In this work, we employed MCC950 as a positive control in in vivo experiments to confirm the effects of Gyp LXXV or Rg3 on improving efficacy of hepatic 
fibrosis and inflammation in the MCD-fed mouse model. In addition, pioglitazone is currently being used in NASH patients with type 2 diabetes, which improves liver injury and fibrosis. Ezetimibe, a lipid-lowering agent, has been reported to improve hepatic steatosis, inflammation, and fibrosis [33]. Accordingly, pioglitazone, ezetimibe, or MCC95 were used as positive controls in this work.

\subsection{Gyp LXXV Inhibits the TGF- $\beta$-Induced Activation of Hepatocytes (HepG2 Cells)}

We performed the cell viability assay of the compounds using hepatocytes, HepG2 cells. As presented in Supplementary Information (Figure S2), an alleviated effect against a palmitate-induced cell death was significantly enhanced by either administration of Rg3 or Gyp LXXV in the cells.

\subsection{Inflammasome Activity in Hepatic Macrophages (THP-1 Cells)}

Hepatic macrophages play an important role in keeping hepatic immune homeostasis [34-36]. Their chief function in the pathogenesis of NASH has made them an attractive therapeutic target for NASH treatment $[35,36]$. To examine the potential effect of macrophage-mediated inflammation in NASH, the hepatic macrophages, THP-1, were treated with LPS + ATP to induce an inflammasome activity. Then, the Gyp LXXV or Rg3 compounds were treated on the THP-1 cells. While the stimulation of THP-1 with LPS and ATP increased secretion of IL-1 $\beta$ (Figure $2 a$ ) or TNF- $\alpha$ (Figure 2b,c), the treatment of Rg3 or Gyp LXXV substantially attenuated the secretion levels, indicating that the compounds could inhibit the activation of inflammasome. Particularly, $10 \mathrm{ng} / \mathrm{mL}$ of Gyp LXXV substantially attenuated the activation level of LPS + ATP-stimulated TNF- $\alpha$ that was increased at the higher concentration of the compound.

(a)

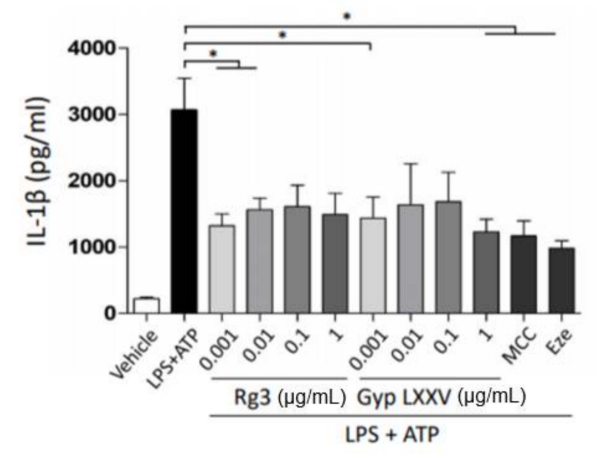

(b)

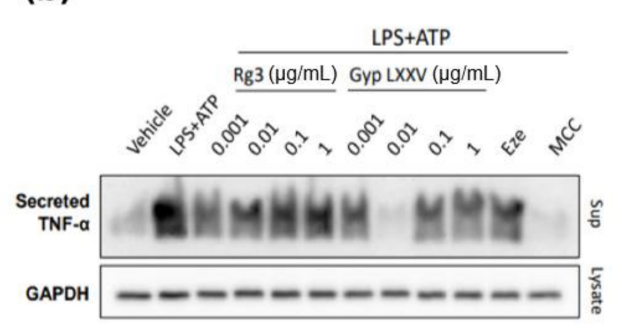

(c)

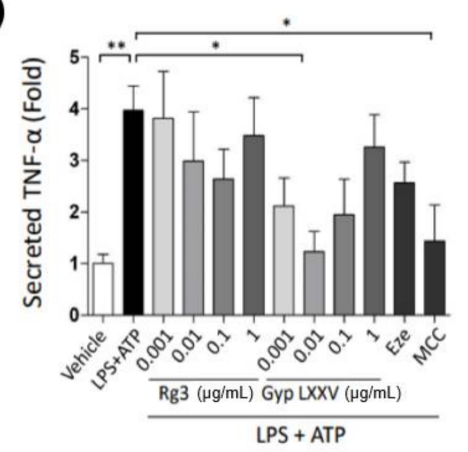

Figure 2. Inhibition effect of Gyp LXXV on inflammation using macrophage-induced THP-1 cells. Attenuated secretion level of IL-1 $\beta$ (a) or TNF- $\alpha(\mathbf{b}, \mathbf{c})$ for Gyp LXXV or Rg3 at various concentration (0.001-1 $\mu \mathrm{g} / \mathrm{mL})$. THP-1 cells were stimulated with lipopolysaccharide (LPS) $(0.1 \mu \mathrm{g} / \mathrm{mL})$ and ATP $(2 \mathrm{mM}) .{ }^{*} p<0.05$ vs. LPS + ATP group. MCC $(10 \mathrm{nM})$ or ezetimibe $($ Eze; $50 \mu \mathrm{M})$ was used as a positive control.

There are many reports that NOD-like receptor protein 3 (NLRP3) inflammasome activation in NAFLD causes liver inflammation [37-39]. However, to better understand macrophage cells to develop 
novel macrophage-based therapeutic interventions in hepatic inflammation, more research on their phenotypes and functions is required. To further confirm the pro-inflammatory profile in hepatic macrophages, we investigated the effects of Gyp LXXV or Rg3 on the mRNA levels of pro-inflammatory genes induced by LPS in THP-1 cells (Figure S3). The LPS-induced TNF- $\alpha$ expression was reduced by Gyp LXXV or Rg3 treatment, while IL-10 expression was unchanged.

\subsection{Effects of Gyp LXXV on Inflammation and Endoplasmic Reticulum (ER) Stress in Hepatocytes (HepG2 Cells)}

To examine an inhibitory effect of inflammation and ER stress in hepatocytes, HepG2 cells were induced with palmitate $(0.01,0.1,1,10 \mu \mathrm{g} / \mathrm{mL})$ and treated with the Gyp LXXV or Rg3 compounds onto the cells. It was confirmed that both compounds exhibited noticeable inhibition effect against TNF- $\alpha$ from the palmitate-induced cells, while showing slight inhibitory effect against GRP78 from the cells (Figure S4).

\subsection{Effect of Gyp LXXV on NASH in Methionine- and Choline-Deficient (MCD) Diet-Induced Mice}

The MCD diet is a common reproducible model to gradually induce the phenotype of severe $\mathrm{NASH}$, including steatosis, hepatic inflammation, and fibrosis after administration, which induces NAFLD/NASH, causing histologically close liver damage to human NASH [40].

To evaluate the effect of Gyp LXXV on non-alcoholic steatohepatitis (NASH), we fed mice with the methionine- and choline-deficient (MCD) diet for 6 weeks. After oral administration of the compounds, the body weight of mice was measured every week. There was no weight change in groups of Gyp LXXV as well as Rg3 compared to the MCD diet mouse group that showed a significant decrease in weight (Figure S5). It was confirmed that the liver tissue/weight ratio of the group administered with each compound was significantly reduced compared to the MCD group (Figure 3a,c). The serum levels of alanine aminotransferase (ALT) and aspartate aminotransferase (AST) are surrogate markers for liver injury. When Rg3 was administered in the MCD diet model, the serum levels of ALT and AST were confirmed to be comparatively unchanged compared to the MCD group (Figure 3b). When Gyp LXXV was fed, the levels were not changed compared to the MCD group, excluding the increased level $(<\sim 800 \mathrm{U} / \mathrm{L})$ in the group of $60 \mathrm{mg} / \mathrm{kg}$ dosed (Figure 3d), which is unharmed since a hepatotoxicity is defined as AST or ALT concentrations greater than $1000 \mathrm{IU} / \mathrm{L}$.

A histological study also provided evidence supporting the results of the serum biochemical analysis. As shown in Figure 4, MCD diet-treated mice had obvious vesicular lesions and severe neutrophil infiltration. In animals treated with MCC950, greatly reduced fat droplets were exhibited when compared to the free MCD model. Consistently, Gyp LXXV and Rg3 displayed significant reductions of fat droplets compared to the free MCD model. Extraordinarily, at an equal level of a low dose range $(15 \mathrm{mg} / \mathrm{kg})$, the reduction rate of fat accumulation in the Gyp LXXV-treated group (Figure 4a) was obviously greater than that of the Rg3-treated group (Figure $4 b$ ), which allows prevention of MCD diet-induced liver injury and lipid accumulation. Moreover, this meets the preferred condition, meaning that it is safe to pursue low dosage-based administration to avoid any potential hepato-cytotoxicity and side effects when compared with results elsewhere on significantly high oral doses of Gyp extracts $(200-800 \mathrm{mg} / \mathrm{kg})$ to treat T2DM-NAFLD [30].

To confirm the lipid accumulation in the liver of MCD-fed mice, oil red O staining was used to measure fat loading in the hepatocytes (Figure 5a). Histological analysis revealed an increased intracellular lipid deposition in the liver of MCD group. The lipid droplets were markedly reduced in the livers of Rg3- or Gyp LXXV-treated mice. In particular, the reduction rate of fat accumulation in the Gyp LXXV-treated group at a low dose range $(15 \mathrm{mg} / \mathrm{kg})$ was apparently greater than that of the $\mathrm{Rg} 3$-treated group, as quantitated in Figure $5 \mathrm{~b}$. We have also monitored the level of lipid metabolic parameters such as triglycerides (TG), which remained unchanged (Figure S6). It is assumed that Gyp LXXV may be effective in other lipid types in NASH-associated lipid metabolism other than TG. 
(a)

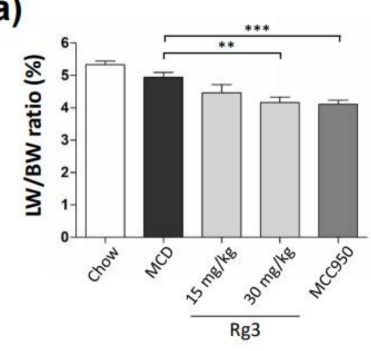

(c)

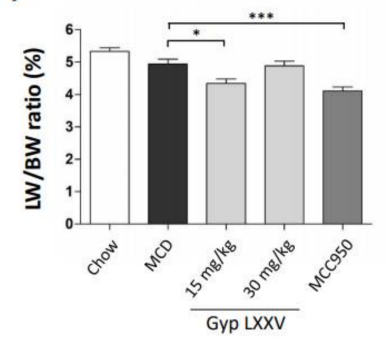

(b)

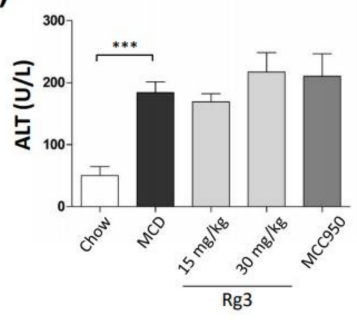

(d)

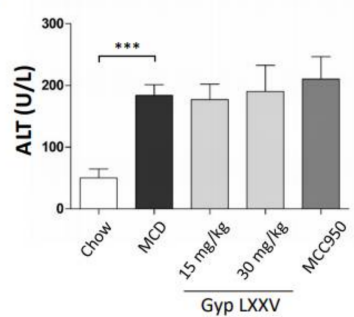

Figure 3. Effect of $\operatorname{Rg} 3(\mathbf{a}, \mathbf{b})$ or Gyp LXXV (c,d) on the liver-to-body weight ratios (LW/BW ratio) and serum enzyme levels (ALT) in the MCD diet mouse model. Error bars represent SEM. ${ }^{*} p<0.05,{ }^{* *} p<$ $0.01,{ }^{* * *} p<0.001$ vs. the MCD group, two tailed unpaired t-test.
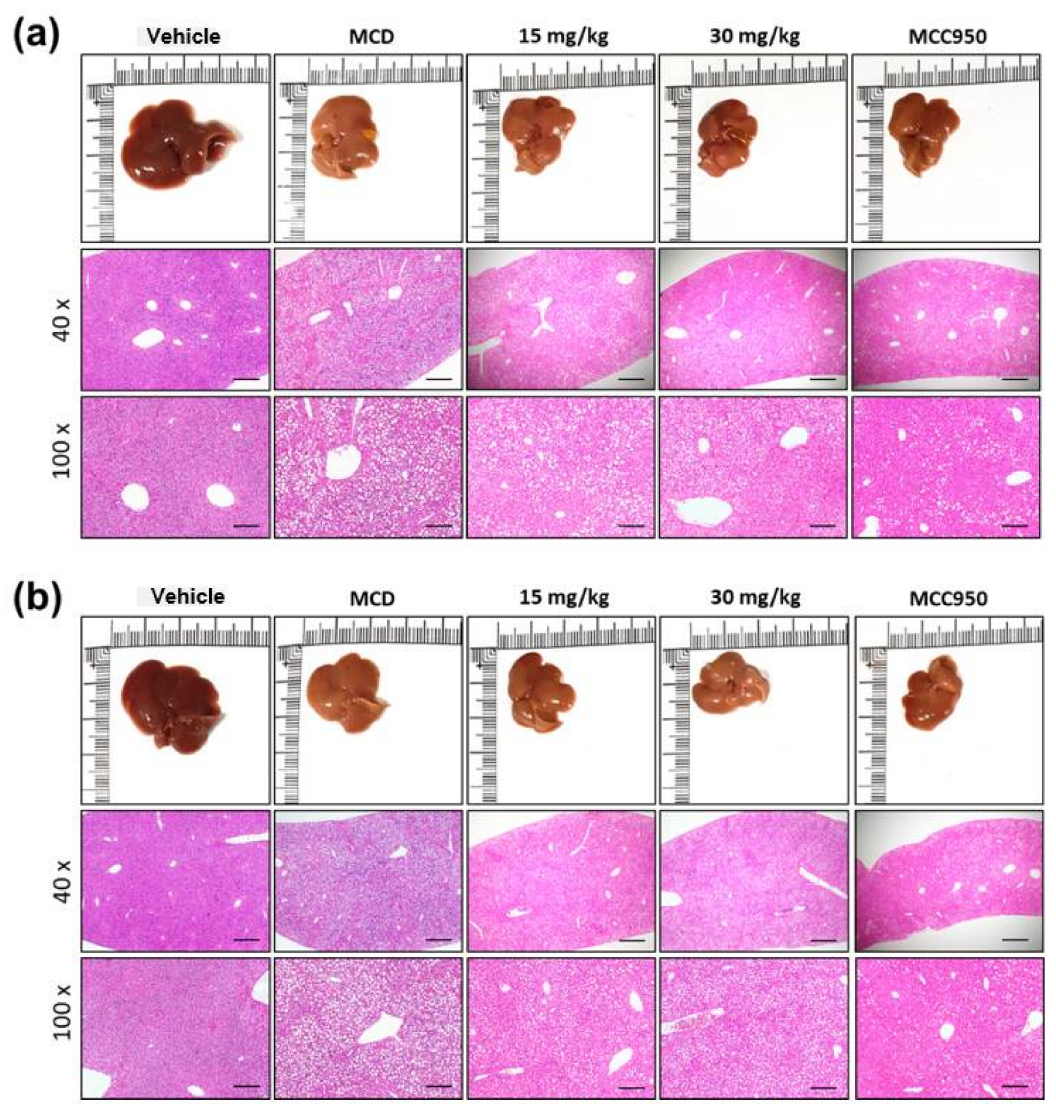

Figure 4. Alleviating effect of Rg3 or Gyp LXXV on fatty liver and hepatitis in the MCD diet mouse model. Representative photographs of the fresh livers and histopathological haematoxylin eosin (H\&E) staining of liver sections administered Rg3 (a) or Gyp LXXV (b) (15-30 mg/kg) are presented the middle panels with a $40 \times$ magnification and a scale bar $500 \mu \mathrm{m}$, and the lower panels with a $100 \times$ magnification and a scale bar $200 \mu \mathrm{m}$. 
(a)
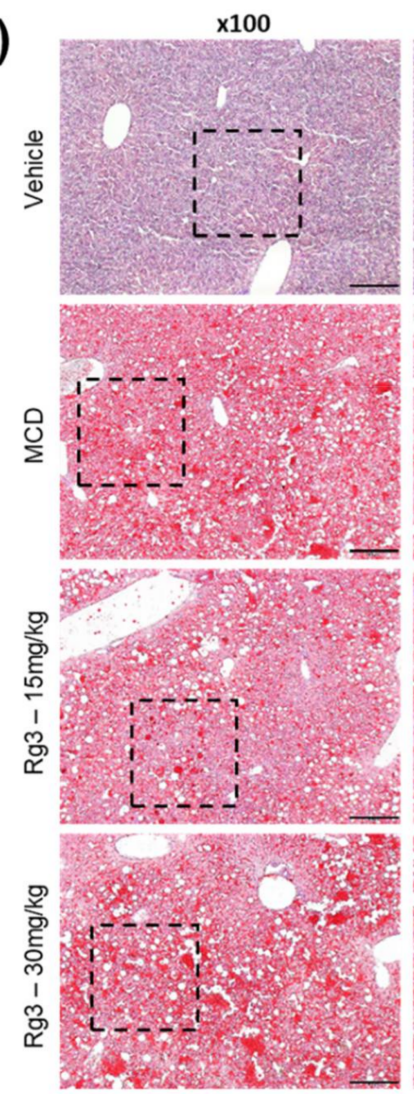

Enlarged
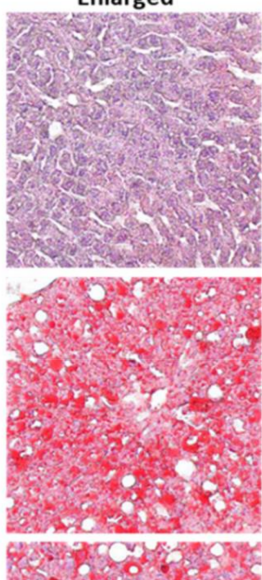

밈
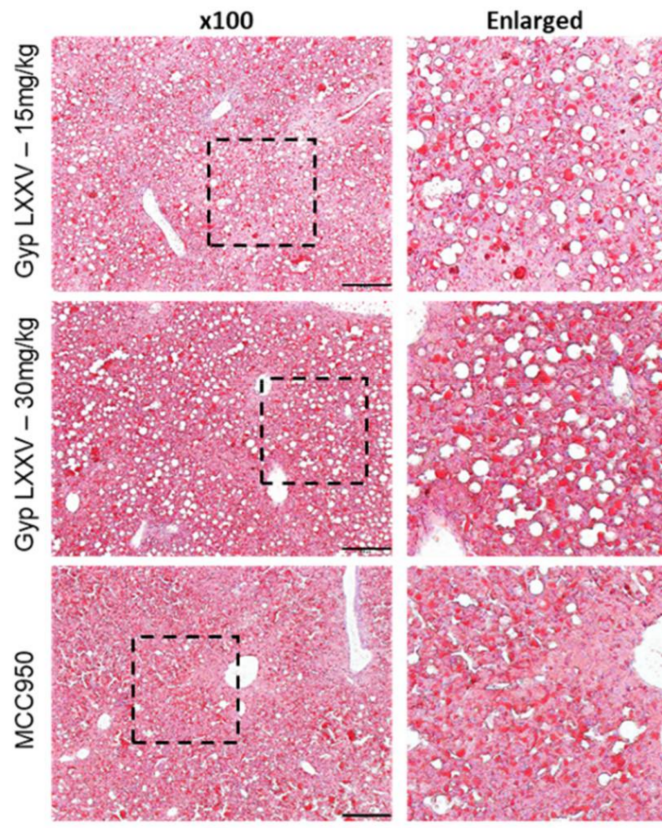

(b)

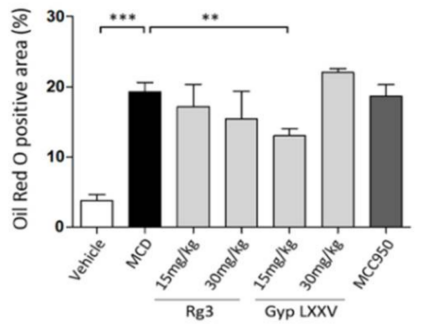

Figure 5. Oil red $\mathrm{O}$ staining of liver sections from the control and MCD-induced mice groups to validate the ameliorating effect of Gyp LXXV against hepatic lipid accumulation. (a) 100x magnification, scale bar $200 \mu \mathrm{m}$. Quantification of oil red O staining was performed using Fiji software (b). Error bars represent SEM. ${ }^{* *} p<0.01,{ }^{* * *} p<0.001$ vs. MCD, two tailed unpaired t-test.

\subsection{Effect of Gyp LXXV in Hepatic Fibrosis in MCD Diet-Induced Mice}

Hepatic fibrosis is an advanced liver disease state that could develop into hepatocellular carcinoma, characterized by the net accumulation of extracellular matrix resulting from NASH $[9,14,15]$. However, there is no direct approved anti-fibrotic therapy, and existing treatment is mostly the invasive elimination of the associated factors, e.g., surgery or transplantation [14].

To confirm whether Gyp LXXV suppressed the activation of hepatic fibrosis in HSCs in vivo, we performed immunohistochemistry (IHC) for $\alpha$-smooth muscle actin ( $\alpha$-SMA), a well-characterized fibrosis marker. As presented in IHC images (Figure 6a) and the quantitative data of $\alpha$-SMA (Figure 6b), the distribution of hepatic fibrosis was substantially reduced in mice treated with Gyp LXXV compared to the group treated with $\mathrm{Rg} 3$, as well as mice fed MCD diet. 
(a)

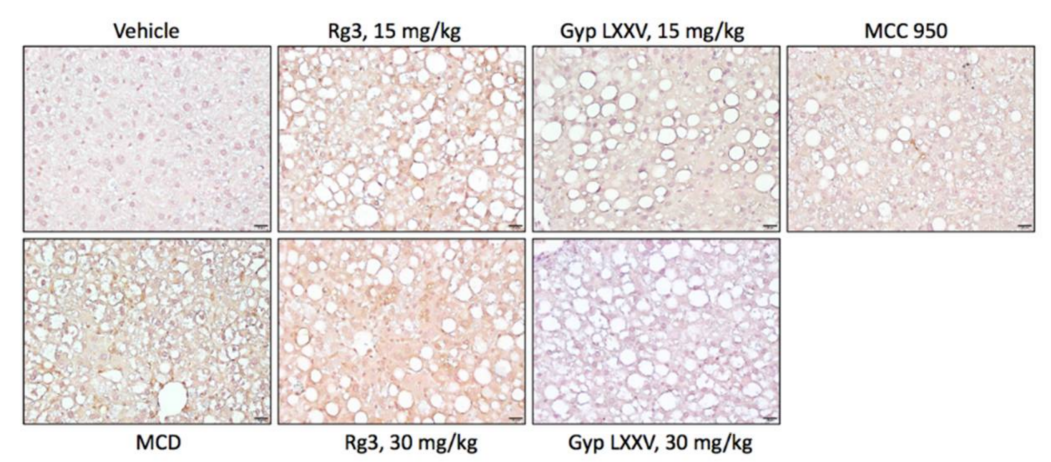

(b)

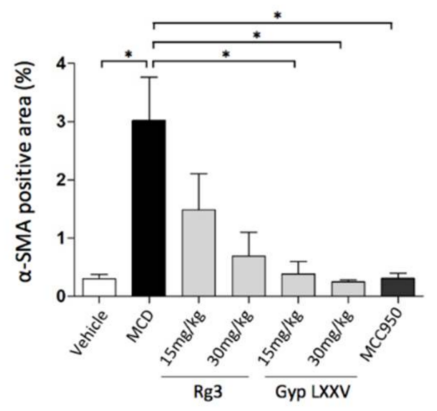

Figure 6. Immunohistochemical staining of $\alpha$-SMA in liver samples (a). The distribution of hepatic fibrosis in the MCD diet mouse model with or without Gyp LXXV or Rg3 was investigated. $400 \times$ magnification, scale bar $20 \mu \mathrm{m}$. Quantification of immunohistochemistry (IHC) for $\alpha$-SMA was performed using Fiji software (b). Error bars represent SEM. ${ }^{*} p<0.05$ vs. MCD, two tailed unpaired t-test.

We also examined the hepatic mRNA expression of activation markers. As presented in Figure 7a, Western blot analysis revealed that the MCD group showed a more increased $\alpha$-SMA expression than the vehicle group, while this level was significantly reduced by administration of Gyp LXXV $(15 \mathrm{mg} / \mathrm{kg})$. The mRNA expression of $\alpha$-SMA and Colla1 were reduced in mice treated with $15 \mathrm{mg} / \mathrm{kg}$ Gyp LXXV compared to mice fed MCD diet, but it was not statistically significant (Figure 7b). As shown in Figure 7c, the administration of $15 \mathrm{mg} / \mathrm{kg}$ Gyp LXXV remarkably reduced the mRNA expression of TGF- $\beta 1$ to a level similar to the vehicle levels in the MCD-fed mouse model while exhibiting no change in the expression levels of TNF- $\alpha$, MCP-1, and IL-1 $\beta$ (Figure 7d-f). Remarkably, treatment with $15 \mathrm{mg} / \mathrm{kg}$ of Gyp LXXV resulted in substantial declines in the expression level of all the markers, corresponding to the H\&E staining results of the liver sections. Henceforth, these data imply that a low dose of Gyp LXXV can inhibit hepatic fibrosis in vivo. Notably, an increase in TGF- $\beta 1$ expression is considered as a key driver in HSC activation to aggravate liver fibrosis [16,17]. As presented in Figure $7 c$, the expression of the TGF- $\beta 1$ marker was prominently induced in the mouse model of liver fibrosis. The oral administration of Gyp LXXV significantly reduced the upregulation of hepatic TGF- $\beta 1$ proteins. 
(a)
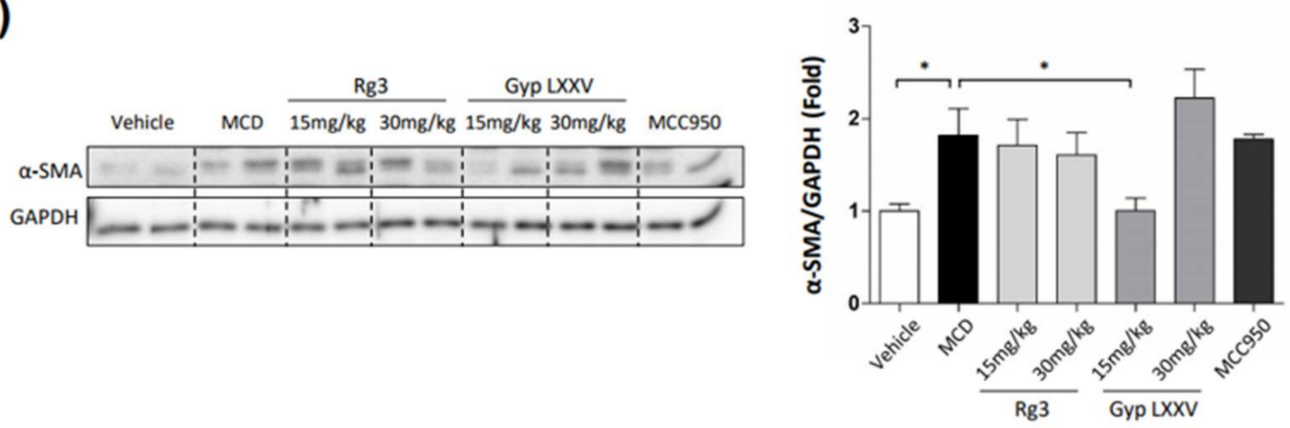

(b)

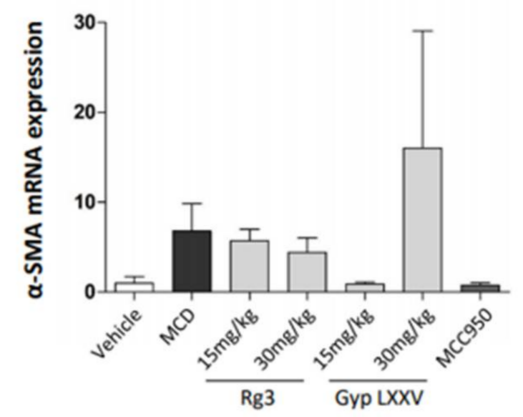

(c)
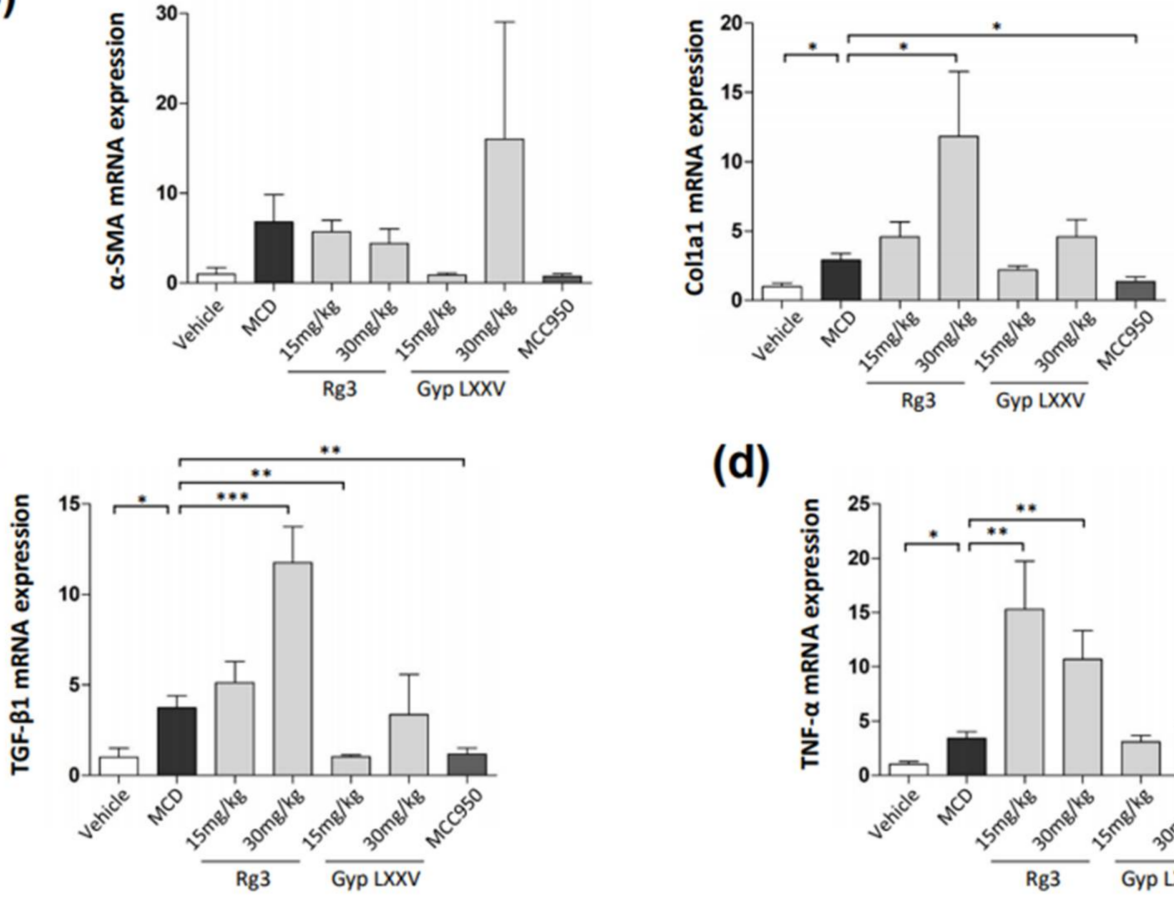

(d)

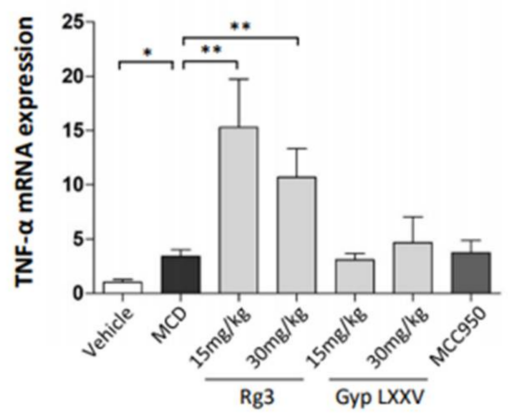

(e)

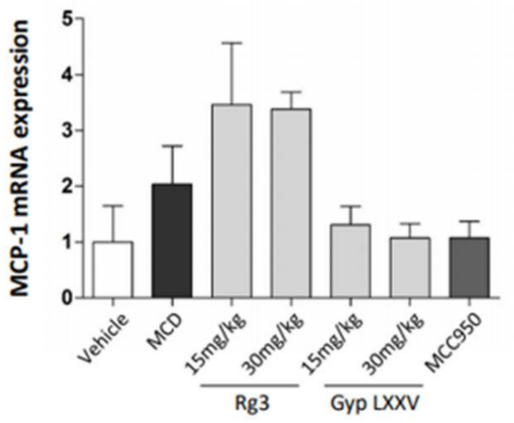

(f)

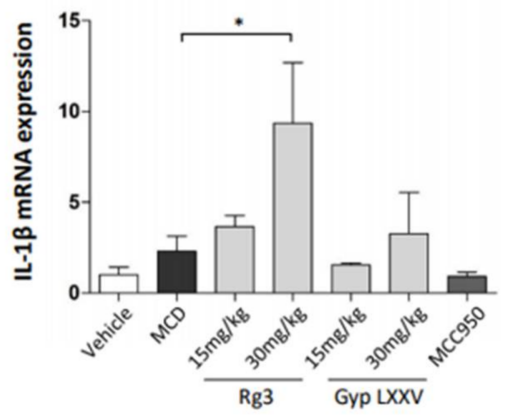

Figure 7. Inhibitory effect of Gyp LXXV on hepatic fibrosis in the MCD diet mouse model. The Western blot analysis of the liver tissues (left) to quantify $\alpha$-SMA/GAPDH (right) (a). Table 1 a1, TGF- $\beta 1$, TNF- $\alpha$, MCP-1, and IL-1 $\beta$ in liver tissues were examined using a RT-qPCR $(\mathbf{b}-\mathbf{f})$. MCC950 $(20 \mathrm{mg} / \mathrm{kg})$ was used as a positive control. The error bars represent SEM. ${ }^{*} p<0.05,{ }^{* *} p<0.01,{ }^{* * *} p<0.001$ vs. MCD, two tailed unpaired t-test.

\subsection{Effect of Gyp LXXV on the Activation of Liver Macrophages in MCD Diet Models}

Macrophages are among the main inflammatory cells that play a role in inducing hepatitis [34]. Subsequent activation of Kupffer cells (KCs) and inflammasomes promotes massive release of 
pro-inflammatory, pro-fibrotic cytokines and ligands. The HSC is then stimulated to produce a large amount of extracellular matrix that causes progressive fibrosis [35]. The effect of Gyp LXXV on the activation of KCs in the liver was confirmed in the MCD mouse model through liver tissue F4/80 staining (Figure 8a). Compared to the MCD model, the quantitative effect of alleviating KC accumulation in the liver was observed in all the Gyp LXXV- and Rg3-treated groups (Figure 8b). Nuclear factor $\kappa \mathrm{B}(\mathrm{NF} \kappa \mathrm{B})$ in macrophages could lead to liver inflammation and fibrosis, which regulates the inflammatory state and plays a key role in immune response [35]. Consistently with the IHC results, our Western blot analysis results have revealed that the level of $\mathrm{p}-\mathrm{NF} \kappa \mathrm{B}$ expression of the group fed with either Rg3 or Gyp LXXV was significantly reduced compared to the MCD-fed group (Figure 8c), suggesting that the compounds may be an effective regulatory pathway into the activation towards liver fibrosis, enabling protection of NASH.

(a)

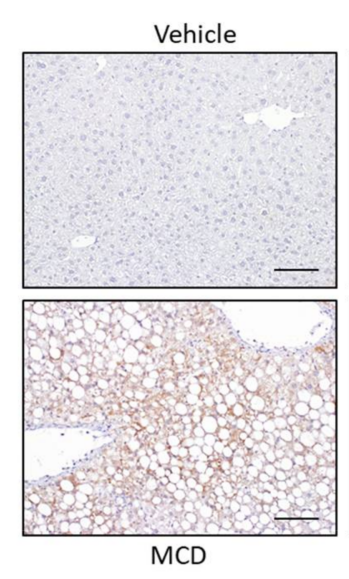

(b)

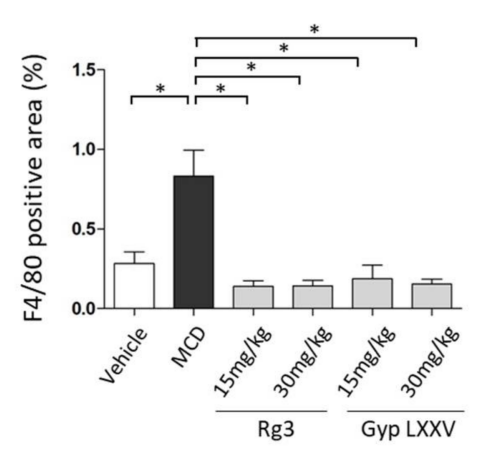

$\mathrm{Rg} 3,15 \mathrm{mg} / \mathrm{kg}$
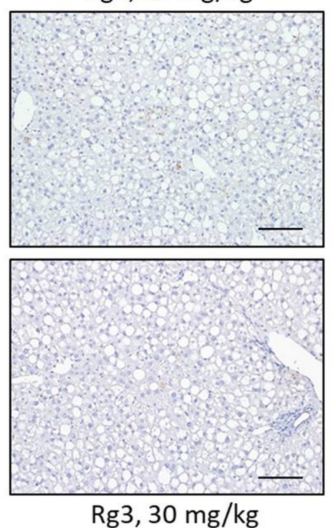

(c)
Gyp LXXV, 15 mg/kg

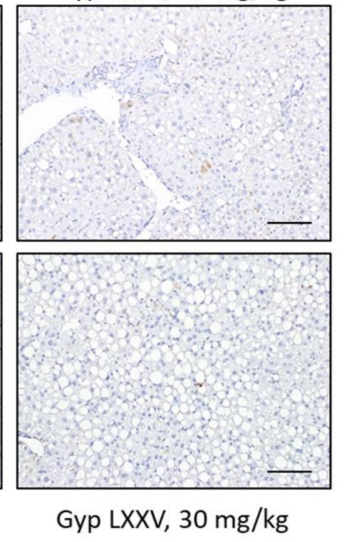

Figure 8. Inhibitory effect of Gyp LXXV on hepatic macrophages in the MCD diet mouse model. Representative IHC staining for F4/80 in the liver (a). The distribution of intracellular hepatic macrophages in the MCD diet mouse model with or without Gyp LXXV or Rg3 was investigated. $200 \times$ magnification, scale bar $100 \mu \mathrm{m}$. Quantification of F4/80 positive cells in paraffin sections of the liver (b). Error bar represent SEM. ${ }^{*} p<0.05$ vs. MCD group, two tailed unpaired t-test. The protein levels of $\mathrm{p}-\mathrm{NF} \kappa \mathrm{B}$ and nuclear factor $\kappa \mathrm{B}(\mathrm{NF} \kappa \mathrm{B})$ were determined by Western blot analysis $(\mathrm{c})$.

Taken together, the molecular inhibition mechanism of NASH signaling pathway by Gyp LXXV molecules is proposed in Figure 9. The hepatocyte injury by fat accumulation activates macrophages and HSCs through some cytokines (TGF- $\beta 1$, IL- $1 \beta$, and TNF- $\alpha$ ), NFKB, and ER stress markers (GRP78), along with proliferation of fibrogenic cells by the activated HSCs in the presence of $\alpha$-SMA, collagen1, TGF- $\beta 1$, TNF- $\alpha$, MCP-1, and IL-1 $\beta$. Gyp LXXV compounds alleviate hepatic injury, inflammation, and fibrosis by down-regulating the hepatic fibrosis pathway, enabling a potential anti-NASH drug. 


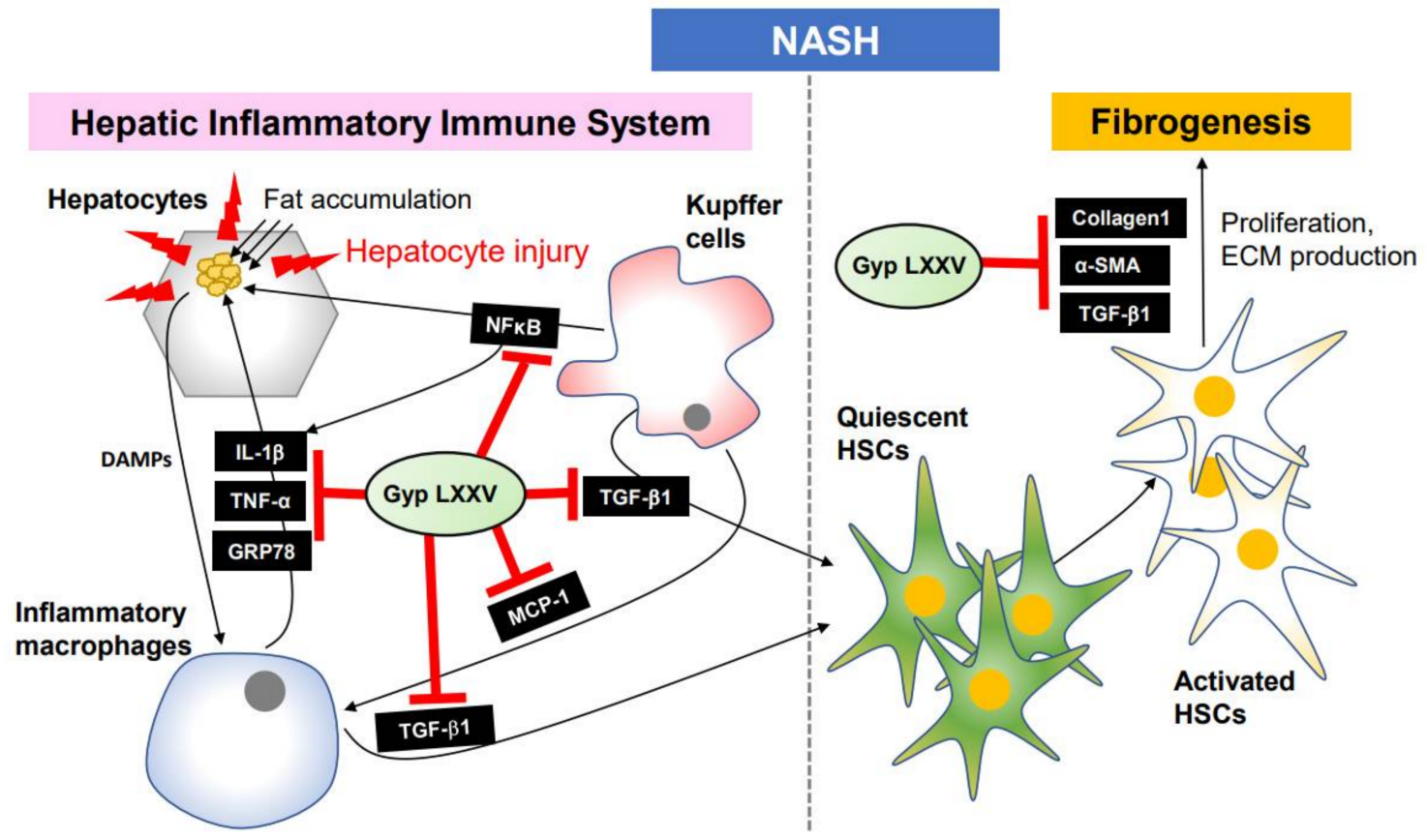

Figure 9. Schematic hepatic inflammation-dependent regulatory mechanism of Gyp LXXV against non-alcoholic steatohepatitis (NASH).

\section{Conclusions}

NASH is characterized by hepatocellular injury accompanied by steatosis, inflammation, and fibrosis, which should be targeted by modulating the signaling pathway to prevent and treat liver fibrosis as early as possible prior to further progression. In this study, Gyp LXXV, a natural ginseng extract-based potent small-molecule inhibitor, efficiently reduces liver lipid accumulation in MCD diet-induced mice. Moreover, it inhibited the progression of liver fibrosis by suppressing the activation of the fibrogenesis signaling pathway by blocking regulatory proteins to lessen liver fibrosis, implying that it is a novel and potential therapeutic strategy for antifibrotic intervention of NASH. Although the efficacy of the compounds was superior to the control groups, it was not concentration dependent. To move onto the clinical trials, further studies such as pharmacokinetics, pharmacodynamics, and safety remain to be explored.

Supplementary Materials: The following are available online at http://www.mdpi.com/2218-273X/10/10/1426/s1, Figures S1-S6.

Author Contributions: Conceptualization: J.-H.K., J.H.L., and Y.-h.L. Data curation and analysis: J.Y.O., J.-H.K., S.H.K., I.J.O., and K.W.L. Project administration: J.-H.K. and Y.-h.L. Funding acquisition: J.H.L. and W.H.L. Writing of original draft: J.-H.K. Writing, review, and editing: J.-H.K., Y.-h.L., and J.Y.O. All authors have read and agreed to the published version of the manuscript.

Funding: This research received no external funding.

Conflicts of Interest: The authors declare no conflict of interest.

\section{References}

1. Parthasarathy, G.; Revelo, X.; Malhi, H. Pathogenesis of Nonalcoholic Steatohepatitis: An Overview. Hepatol Commun. 2020, 4, 478-492. [CrossRef]

2. Ignat, S.R.; Dinescu, S.; Hermenean, A.; Costache, M. Cellular Interplay as a Consequence of Inflammatory Signals Leading to Liver Fibrosis Development. Cells 2020, 9, 461. [CrossRef]

3. Schuster, S.; Cabrera, D.; Arrese, M.; Feldstein, A.E. Triggering and resolution of inflammation in NASH. Nat. Rev. Gastroenterol. Hepatol. 2018, 15, 349-364. [CrossRef] [PubMed] 
4. Veal, J.; Mcbride, C.; Lazic, M.; Povero, D.; Ambrus, G.; Santini, A.; Stansfield, R.; Trzoss, L.; Johnson, C.D.; Stafford, J.; et al. Discovery of Inhibitors of NLRP3 inflammasome assembly for the treatment of NASH and liver fibrosis. J. Hepatol. 2018, 68, S347-S348. [CrossRef]

5. Wan, X.; Xu, C.; Yu, C.; Li, Y. Role of NLRP3 Inflammasome in the Progression of NAFLD to NASH. Can J. Gastroenterol. Hepatol. 2016, 2016, 6489012. [CrossRef] [PubMed]

6. Ganz, M.; Szabo, G. Immune and inflammatory pathways in NASH. Hepatol. Int. 2013, 7 (Suppl. 2), 771-781. [CrossRef] [PubMed]

7. Pan, J.; Ou, Z.; Cai, C.; Li, P.; Gong, J.; Ruan, X.Z.; He, K. Fatty acid activates NLRP3 inflammasomes in mouse Kupffer cells through mitochondrial DNA release. Cell Immunol. 2018, 332, 111120. [CrossRef]

8. Jia, Y.; Ji, P.; Nguyen, L.; French, B.; Tillman, B.; French, S.W. Different Roles of Epigenetic Regulators and Inflammasome in Hepatocellular Carcinoma Tumorigenesis in Patients with ASH vs. NASH. FASEB J. 2019, $33,662.67$.

9. Sircana, A.; Paschetta, E.; Saba, F.; Molinaro, F.; Musso, G. Recent Insight into the Role of Fibrosis in Nonalcoholic Steatohepatitis-Related Hepatocellular Carcinoma. Int. J. Mol. Sci. 2019, 20, 1745. [CrossRef]

10. Yoon, H.-J.; Lee, Y.-H.; Cha, B.-S. Causal Relationship of Non-alcoholic Fatty Liver Disease with Obesity and Insulin Resistance. J. Korean Diabetes. 2014, 15, 76-81. [CrossRef]

11. Bhatt, H.B.; Smith, R.J. Fatty liver disease in diabetes mellitus. Hepatobiliary Surg. Nutr. 2015, 4, 101-108. [PubMed]

12. Léveillé, M.; Estall, J.L. Mitochondrial Dysfunction in the Transition from NASH to HCC. Metabolites 2019, 9, 233. [CrossRef] [PubMed]

13. Peverill, W.; Powell, L.W.; Skoien, R. Evolving concepts in the pathogenesis of NASH: Beyond steatosis and inflammation. Int. J. Mol. Sci. 2014, 15, 8591-8638. [CrossRef] [PubMed]

14. Wattacheril, J.; Issa, D.; Sanyal, A. Nonalcoholic Steatohepatitis (NASH) and Hepatic Fibrosis: Emerging Therapies. Annu. Rev. Pharmacol. Toxicol. 2018, 58, 649-662. [CrossRef]

15. Zhang, C.-Y.; Yuan, W.-G.; He, P.; Lei, J.-H.; Wang, C.-X. Liver fibrosis and hepatic stellate cells: Etiology, pathological hallmarks and therapeutic targets. World J. Gastroenterol. 2016, 22, 10512-10522. [CrossRef]

16. Yang, L.; Roh, Y.S.; Song, J.; Zhang, B.; Liu, C.; Loomba, R.; Seki, E. Transforming growth factor beta signaling in hepatocytes participates in steatohepatitis through regulation of cell death and lipid metabolism in mice. Hepatology 2014, 59, 483-495. [CrossRef]

17. Yokoyama,H.; Masaki, M.; Inoue, I.; Nakamura, M.; Mezaki, Y.; Saeki, C.; Oikawa, T.; Saruta, M.; Takahashi,H.; Ikegami, M.; et al. Histological and biochemical evaluation of transforming growth factor- $\beta$ activation and its clinical significance in patients with chronic liver disease. Heliyon 2019, 5, e01231. [CrossRef]

18. Gawrieh, S.; Chalasani, N. Emerging Treatments for Nonalcoholic Fatty Liver Disease and Nonalcoholic Steatohepatitis. Clin. Liver Dis. 2018, 22, 189-199. [CrossRef]

19. Glass, O.; Filozof, C.; Noureddin, M.; Berner-Hansen, M.; Schabel, E.; Omokaro, S.O.; Schattenberg, J.M.; Barradas, K.; Miller, V.; Francque, S.; et al. Standardisation of diet and exercise in clinical trials of NAFLD-NASH: Recommendations from the Liver Forum. J. Hepatol. 2020, 73, 680-693. [CrossRef]

20. Kim, J.H.; Yi, Y.-S.; Kim, M.-Y.; Cho, J.Y. Role of ginsenosides, the main active components of Panax ginseng, in inflammatory responses and diseases. J. Ginseng Res. 2017, 41, 435-443. [CrossRef]

21. Lee, H.; Kong, G.; Tran, Q.; Kim, C.; Park, J.; Park, J. Relationship Between Ginsenoside Rg3 and Metabolic Syndrome. Front Pharmacol. 2020, 11, 130. [CrossRef] [PubMed]

22. Lee, H.U.; Bae, E.A.; Han, M.J.; Kim, D.H. Hepatoprotective effect of 20(S)-ginsenosides Rg3 and its metabolite 20(S)-ginsenoside Rh2 on tert-butyl hydroperoxide-induced liver injury. Biol. Pharm. Bull. 2005, 28, 1992-1994. [CrossRef] [PubMed]

23. Kim, J.C.; Jeon, J.Y.; Yang, W.S.; Kim, C.H.; Eom, D.W. Combined amelioration of ginsenoside (Rg1, Rb1, and $\mathrm{Rg} 3$ )-enriched Korean red ginseng and probiotic lactobacillus on non-alcoholic fatty liver disease. Curr. Pharm. Biotechnol. 2019, 20, 222-231. [CrossRef] [PubMed]

24. Xiao, Q.; Zhang, S.; Yang, C.; Du, R.; Zhao, J.; Li, J.; Xu, Y.; Qin, Y.; Gao, Y.; Huang, W. Ginsenoside Rg1 Ameliorates Palmitic Acid-Induced Hepatic Steatosis and Inflammation in HepG2 Cells via the AMPK/NF-kB Pathway. Int. J. Endocrinol. 2019, 2019, 7514802. [CrossRef]

25. Chen, D.-J.; Hu, H.-G.; Xing, S.-F.; Gao, Y.-J.; Xu, S.-F.; Piao, X.-L. Metabolic profiling of Gynostemma pentaphyllum extract in rat serum, urine and faeces after oral administration. J. Chromatogr. B 2014, 969, 42-52. [CrossRef] 
26. Wang, J.; Yang, J.-L.; Zhou, P.-P.; Meng, X.-H.; Shi, Y.-P. Further new gypenosides from jiaogulan (Gynostemma pentaphyllum). J. Agr. Food Chem. 2017, 65, 5926-5934. [CrossRef]

27. Park, S.; Ko, E.; Lee, J.H.; Song, Y.; Cui, C.-H.; Hou, J.; Jeon, B.M.; Kim, H.S.; Kim, S.C. Gypenoside LXXV Promotes Cutaneous Wound Healing In Vivo by Enhancing Connective Tissue Growth Factor Levels Via the Glucocorticoid Receptor Pathway. Molecules 2019, 24, 1595. [CrossRef]

28. Li, H.; Ying, H.; Hu, A.; Hu, Y.; Li, D. Therapeutic Effect of Gypenosides on Nonalcoholic Steatohepatitis via Regulating Hepatic Lipogenesis and Fatty Acid Oxidation, Biol. Pharm. Bull. 2017, 40, 650-657. [CrossRef]

29. Huang, X.; Chen, W.; Yan, C.; Yang, R.; Chen, Q.; Xu, H.; Huang, Y. Gypenosides improve the intestinal microbiota of non-alcoholic fatty liver in mice and alleviate its progression. BioMed. Pharmacother. 2019, 118, 109258. [CrossRef]

30. He, Q.; Li, J.K.; Li, F.; Li, R.G.; Zhan, G.Q.; Li, G.; Du, W.X.; Tan, H.B. Mechanism of action of gypenosides on type 2 diabetes and non-alcoholic fatty liver disease in rats. World J. Gastroentero 2015, 21, 2058-2066. [CrossRef]

31. Lee, K.W.; Lee, W.H.; Han, B.-S.; Lee, J.H.; Doo, E.K.; Kim, J.-H. Molecular Drug Discovery of Single Ginsenoside Compounds as a Potent Bruton's Tyrosine Kinase Inhibitor. Int. J. Mol. Sci. 2020, 21, 3065. [CrossRef] [PubMed]

32. Mridha, A.R.; Wree, A.; Farrell, G.C. NLRP3 inflammasome blockage reduces liver inflammation and fibrosis in experimental NASH in mice. J. Hepatology. 2017, 66, 1037-1046. [CrossRef] [PubMed]

33. Kim, S.H.; Kim, G.; Lee, M. Ezetimibe ameliorates steatohepatitis via AMP activated protein kinase-TFEB-mediated activation of autophagy and NLRP3 inflammasome inhibiton. Autophagy 2017, 13, 1767-1781. [CrossRef] [PubMed]

34. Heymann, F.; Tacke, F. Immunology in the liver-from homeostasis to disease. Nat. Rev. Gastroenterol. Hepatol. 2016, 13, 88-110. [CrossRef] [PubMed]

35. Tacke, F. Targeting hepatic macrophages to treat liver diseases. J. Hepatol. 2017, 66, 1300-1312. [CrossRef] [PubMed]

36. Lang, D.; Xiaomin, S.; He, X.; Gao, Y. Macrophage Phenotype and Function in Liver Disorder. Front. Immunol. 2020, 10, 3112.

37. Ganz, M.; Cask, T.; Szabo, G. High fat diet feeding results in gender specific steatohepatitis and inflammasome activation. World J. Gastoenterol. 2014, 20, 8525-8534. [CrossRef]

38. Huang, H.; Chen, H.W.; Nace, G.W. Histones activate the NLRP3 inflammasome in Kupper cells during sterile inflammatory liver injury. J. Immunol. 2013, 191, 2665-2679. [CrossRef]

39. Wree, A.; Eguchi, M.D.; Canbay, A. NLRP3 inflammasome activation results in hepatocyte pyroptosis, liver inflammation, and fibrosis in mice. Hepatology 2014, 59, 898-910. [CrossRef]

40. Zamin, I., Jr.; Mattos, A.A.; Mattos, A.Z.; Migon, E.; Soares, E.; Perry, M.L. Model of experimental nonalcoholic steatohepatitis from use of methionine and choline deficient diet. Arq. Gastroenterol. 2009, 46, 69-74. [CrossRef]

(C) 2020 by the authors. Licensee MDPI, Basel, Switzerland. This article is an open access article distributed under the terms and conditions of the Creative Commons Attribution (CC BY) license (http://creativecommons.org/licenses/by/4.0/). 